\title{
Dexmedetomidine hydrochloride inhibits hepatocyte apoptosis and inflammation by activating the IncRNA TUG1/miR-194/SIRT1 signaling pathway
}

Xiao-Xia Gu' ${ }^{1}$ Xiao-Xia Xu ${ }^{2}$,Hui-Hua Liao ${ }^{1}$, Ruo-Na Wu², Wei-Ming Huang ${ }^{3}$, Li-Xia Cheng ${ }^{1}$, Yi-Wen Lu ${ }^{1}$ and Jian $\mathrm{Mo}^{1 *}$

\begin{abstract}
Background: Liver injury seriously threatens the health of people. Meanwhile, dexmedetomidine hydrochloride (DEX) can protect against liver injury. However, the mechanism by which Dex mediates the progression of liver injury remains unclear. Thus, this study aimed to investigate the function of DEX in oxygen and glucose deprivation (OGD)-treated hepatocytes and its underlying mechanism.

Methods: In order to investigate the function of DEX in liver injury, WRL-68 cells were treated with OGD. Cell viability was measured by MTT assay. Cell apoptosis was detected by flow cytometry. Inflammatory cytokines levels were measured by ELISA assay. The interaction between miR-194 and TUG1 or SIRT1 was detected by dualluciferase reporter. Gene and protein levels were measured by qPCR or western blotting.

Results: DEX notably reversed OGD-induced inflammation and apoptosis in WRL-68 cell. Meanwhile, the effect of OGD on TUG1, SIRT1 and miR-194 expression in WRL-68 cells was reversed by DEX treatment. However, TUG1 knockdown or miR-194 overexpression reversed the function of DEX in OGD-treated WRL-68 cells. Moreover, TUG1 could promote the expression of SIRT1 by sponging miR-194. Furthermore, knockdown of TUG1 promoted OGDinduced cell growth inhibition and inflammatory responses, while miR-194 inhibitor or SIRT1 overexpression partially reversed this phenomenon.

Conclusions: DEX could suppress OGD-induced hepatocyte apoptosis and inflammation by mediation of TUG1/ miR-194/SIRT1 axis. Therefore, this study might provide a scientific basis for the application of DEX on liver injury treatment.
\end{abstract}

Keywords: Dexmedetomidine hydrochloride, TUG1, SIRT1, miR-194, liver injury

\footnotetext{
* Correspondence: 343251863@qq.com

'Department of Anesthesiology, Affiliated Hospital of Guangdong Medical University, No.57, South People's Avenue, Xiashan District, 524001 Zhanjiang, Guangdong Province, P.R. China

Full list of author information is available at the end of the article
}

(c) The Author(s). 2021 Open Access This article is licensed under a Creative Commons Attribution 4.0 International License, which permits use, sharing, adaptation, distribution and reproduction in any medium or format, as long as you give appropriate credit to the original author(s) and the source, provide a link to the Creative Commons licence, and indicate if changes were made. The images or other third party material in this article are included in the article's Creative Commons licence, unless indicated otherwise in a credit line to the material. If material is not included in the article's Creative Commons licence and your intended use is not permitted by statutory regulation or exceeds the permitted use, you will need to obtain permission directly from the copyright holder. To view a copy of this licence, visit http://creativecommons.org/licenses/by/4.0/ The Creative Commons Public Domain Dedication waiver (http://creativecommons.org/publicdomain/zero/1.0/) applies to the data made available in this article, unless otherwise stated in a credit line to the data. 


\section{Background}

Liver injury is a common and serious injury in abdominal trauma. Its incidence ranks second only to spleen rupture, and there are many complications of liver injury $[1,2]$. In addition, liver injury usually results from the excessive consumption of alcohol, infections, and xenobiotics, et al. [3]. In addition, it usually leads to liver failure and even death. Nowadays, surgery is the main treatment for liver injury, while the mortality of liver failure has been maintained at a high level [4]. Therefore, it is of great significance to explore the therapeutic targets against liver injury.

Dexmedetomidine hydrochloride (DEX) is a new type of highly selective $\alpha$-adrenergic receptor agonist that has been widely used in the clinic. It was reported that DEX could inhibit endotoxemia-induced inflammatory response and protect against the organ injury [5]. In addition, DEX is known to alleviate the local and systemic inflammation response by mediation of IL- 6 and TNF- $\alpha$ [6]. Furthermore, it had been previously found that DEX could alleviate the severity of hepatic injury [7]. However, the mechanism by which DEX modulates the progression of liver injury remains unclear.

Long non-coding RNAs (lncRNAs) are a class of noncoding RNAs which are over 200 nucleotides in length [8]. A previous study reports that some lncRNAs can play key roles in a variety of diseases, including liver injury [9-11]. In addition, IncRNA taurine up-regulated gene 1 (TUG1) was originally distributed in taurinetreated mouse retinal cells, and it was highly conserved in mammals [12]. A research indicated that TUG1 could regulate the tumor cell growth [13], and TUG1 could attenuate the inflammation during the progression of liver injury [14]. However, the molecular mechanism by which TUG1 attenuates the liver injury is unclear.

MicroRNAs (miRNAs) are a group of small noncoding transcripts which can regulate cell growth and inflammatory responses $[15,16]$. Previous studies have found that miRNAs are involved in liver functions. For example, miR-194 could inhibit hepatic stellate cell activation and extracellular matrix production which were associated with liver fibrosis [17]. miR-194 was found to be up-regulated in liver injury and it could inhibit the activity and fibrosis in hepatic stellate cells [18]. Nevertheless, the function of miR-194 in liver injury is largely unknown.

Sirtuin 1 (SIRT1) is a nicotinamide adenine dinucleotide dependent histone deacetylase which plays a crucial role in gene transcription, oxidative stress and inflammation [19]. SIRT1 was down-regulated in doxorubicin-induced liver injury [20]. Meanwhile, upregulation of SIRT1 could protect against the liver injury [21]. In this study, to the data of bioinformatics indicated TUG1 was bound to miR-194 and SIRT1 was a target gene of miR-194. Consequently, it could be hypothesized that DEX protected the hepatocyte injury though regulating TUG1/miR-194/SIRT1 axis in OGD-induced hepatocytes.

In the present study, the function of DEX in hepatocyte apoptosis and inflammation was investigated. Our findings revealed that DEX could attenuate the liver injury by mediation of TUG1/miR-194/SIRT1 signaling pathway. Our study may be provided a theoretical basis for the treatment of liver injury.

\section{Methods}

\section{Cell culture and drug treatment}

The human hepatocytes (WRL-68) were obtained from American Type Culture Collection (ATCC, Manassas, VA, USA). Cells were cultured in RPMI-1640 with $10 \%$ fetal bovine serum (FBS, Invitrogen, Carlsbad, CA, USA) in a condition of $5 \% \mathrm{CO}_{2}$ and $95 \%$ humidified air at $37{ }^{\circ} \mathrm{C}$. DEX was obtained from Sigma-Aldrich (catalog number: 1,179,333, St Louis, MO, USA) and diluted in $0.1 \%$ dimethyl sulfoxide (DMSO) (Sigma-Aldrich, St. Louis, MO, USA).

\section{Oxygen and glucose deprivation (OGD)-induced liver injury}

WRL-68 cells were pre-treated with different concentrations of DEX $(0,0.01,0.1,1$ or $5 \mathrm{nM}$ DEX) for $1 \mathrm{~h}$. Subsequently, cells were cultured in OGD medium (glucose free and serum-free RPMI-1640) under $37{ }^{\circ} \mathrm{C}$ hypoxia $\left(5 \% \mathrm{CO}_{2}, 1 \% \mathrm{O}_{2}, 94 \% \mathrm{~N}_{2}\right)$ for $4 \mathrm{~h}$ after washed with PBS. Then, cells were reoxygenated $\left(5 \% \mathrm{CO}_{2}, 95 \% \mathrm{O}_{2}\right)$ and replaced by conventional medium for $8 \mathrm{~h}$. Meanwhile, cells in control group were cultured in an incubator with $5 \% \mathrm{CO}_{2}$ at $37^{\circ} \mathrm{C}$.

\section{MTT Assay}

The cells were seeded in 96-well plates at a density of 2000 cells per well. After $24 \mathrm{~h}$ of incubation, the culture medium (200 $\mu \mathrm{L} /$ well) was replaced with FBS-free medium $(200 \mu \mathrm{L} /$ well $)$ containing various concentrations of $\operatorname{DEX}(0.01,0.1,1$ and $5 \mathrm{nM})$. Subsequently, the culture medium was discarded, and $5 \mathrm{mg} / \mathrm{mL} \mathrm{3-(4,5-di-}$ methyl thiazol-2-yl)-5-diphenyltetrazolium bromide (MTT) solutions (Sigma, MO, USA) were added in each well for $3 \mathrm{~h}$ at $37^{\circ} \mathrm{C}$. Then, the cell supernatants were removed and DMSO $(200 \mu \mathrm{L})$ was added. The absorbance of each well was measured at $490 \mathrm{~nm}$ using a microplate reader (Tecan, Mannedorf, Switzerland).

\section{Flow cytometry}

Apoptotic cells were determined using flow cytometry. Cells were plated in 6-well plates at a density of $2 \times 10^{5}$ cells. After incubation of $48 \mathrm{~h}$, cells were collected with trypsin, washed twice with PBS and centrifuged at 1, 
$500 \mathrm{rpm}$ for $5 \mathrm{~min}$. Subsequently, cells were subjected to apoptosis assay with Annexin V-FITC/PI Apoptosis Detection Kit (BD Pharmingen, Franklin Lake, NJ, USA) according to the instructions of manufacturer. Then, cells were analyzed using a BD Biosciences Fluorescence activated Cell Sorting (FACS, BD Pharmingen, Franklin Lake, NJ, USA) Calibur system with CellQuest Pro software [22].

\section{Cell transfection}

The miR-194 inhibitor, miR-194 mimics, negative control (inhibitor/mimics NC), short hairpin-TUG1 (shTUG1) and negative control (sh-NC) were synthetized by Genepharma Inc. (Shanghai, China). For SIRT1 overexpression (pcDNA3.1-SIRT1), the amplified fragment of SIRT1 was inserted into pcDNA3.1 vector (Genepharma Inc., Shanghai, China), and pcDNA3.1 vector was negative control. These plasmids were transfected into cells by using Lipofectamine 2000 (Invitrogen, Carlsbad, CA, USA). Then, cells were cultured with or without DEX for $48 \mathrm{~h}$.

\section{RNA extraction and RT-qPCR analysis}

Total RNA was isolated from cells by using RNA extraction kit (Invitrogen, Carlsbad, CA, USA) according to the manufacturer's protocol. $2 \mu \mathrm{g}$ RNA was synthesized into cDNA using SuperScriptTM IV First-Strand Synthesis System (Invitrogen, USA). Real-time qPCR of the reverse transcription products of TUG1, miR-194 and SIRT1 expression was determined using Permix Ex Taq (Takara, Tokyo, Japan), analyzed through the 7500 Realtime PCR System (Applied Biosystems, USA). The data were quantified by normalizing to GAPDH or U6. Relative expression level of genes was quantified using $2^{-\triangle \Delta C t}$ method. The primer sequences for qPCR were as follows:

miR-194 F: 5'-TGTAACAGCAACTCCATGTG-3', miR-194 R: 5'-GTCGTATCGAGAGCAGGG TCCGAGGTATTCGCACTCGATAC

GACTCCACAT-3',

SIRT1 F: 5'-CAAACTTTGCTGTAACCCTGT-3', SIRT1 R: 5'-CAGCCACTGAAGTTCTTTCAT-3', TUG1 F: 5'-TAGCAGTTCCCCAATCCTTG-3', TUG1 R: 5'-CACAAATTCCCATCATTCCC-3', GAPDH F: 5'-AGGTCGGTGTGAACGGATTTG-3', GAPDH R: 5'- GGGGTCGTTGATGGCAACA-3', U6 F: 5'-CTCGCTTCGGCAGCACAT-3', U6 R: 5'-AACGCTTCACGAATTTGCGT-3'.

Total protein extraction and Western blot analysis

Total protein was isolated from cell lysates by using RIPA buffer. The concentration of protein was detected with a BCA protein kit (Thermo Fisher Scientific, Waltham, MA, USA). Then, proteins $(40 \mu \mathrm{g}$ per lane) were separated with $10 \%$ sodium dodecyl sulfatepolyacrylamide gel electrophoresis (SDS-PAGE) gel and then transferred into polyvinylidene fluoride (PVDF, Thermo Fisher Scientific) membranes. The membrane was then blocked with $5 \%$ nonfat milk dissolved in TBS buffer for $1 \mathrm{~h}$ and incubated with primary antibodies overnight at $4{ }^{\circ} \mathrm{C}$. Subsequently, membranes were incubated with secondary anti-rabbit antibody (1:5000) at room temperature for $1 \mathrm{~h}$. Signals were developed by incubating the membrane with enhanced chemiluminescence and western blot detection reagents, followed by exposure to X-Omat Blue XB-1 film (Kodak, Rochester, NY) for autoradiography. The primary antibodies were as follows: anti-Bax (1:1000), anti-Bcl-2 (1:1000), antiSIRT1 (1:1000) and anti-GAPDH (1:1000). All the antibodies were obtained from Abcam (Cambridge, MA, USA). The data was analyzed by using ImageJ software.

\section{Bioinformatics analysis and dual-luciferase reporter gene assay}

Starbase (http://starbase.sysu.edu.cn) was used to predict the binding sites between miR-194 and TUG1 or SIRT1. The partial sequences of TUG1 and 3'-UTR of SIRT1 containing the putative binding sites of miR-194 were synthetized and obtained from Sangon Biotech (Shanghai, China), and then cloned into the pmirGLO DualLuciferase miRNA Target Expression Vectors (Promega, Madison, WI, USA) for constructing the TUG1 reporter vectors (TUG1-wt/mut) and SIRT1 reporter vectors (SIRT1-wt/mut), respectively. The TUG1-wt/mut vectors or SIRT1-wt/mut vectors were transfected into WRL-68 cells together with mimics NC or miR-194 mimics using Lipofectamine 2000 (Thermo Fisher Scientific). The relative luciferase activity was analyzed by the Dual-Glo Luciferase Assay System (Promega). Renilla luciferase was used as internal control.

\section{ELISA assay}

The levels of IL-1 $\beta$, IL- 6 and TNF- $\alpha$ in the supernatants of WRL-68 cells was determined by ELISA kit (Nanjing Jiancheng Bioengineering Institute, Nanjing, China) according to the instructions of manufacturer. In brief, cells were coated with the primary antibodies overnight, and then blocked with $10 \%$ FBS for $1 \mathrm{~h}$. Subsequently, cells were incubated with the secondary anti-rabbit antibody at room temperature for $1 \mathrm{~h}$. After that, cells were treated with $1 \mathrm{M}$ hydrochloric acid (Beyotime, Shanghai, China). Finally, the OD value was measured by a microreader $(450 \mathrm{~nm}$; Thermo Fisher Scientific, Waltham, MA, USA).

\section{Statistical analysis}

All experiments were conducted in triplicate. Data were expressed as the means \pm standard deviations (SD) and 
analyzed using student's t-test or one-way ANOVA, followed by the Least Significant Difference (LSD) posthoc test. A $p$-value $<0.05$ was considered statistically significant.

\section{Results \\ DEX significantly reversed OGD-induced WRL-68 cell apoptosis and inflammation}

To test the cell viability, MTT assay was performed. As indicated in Fig. 1 a, the survival rate of WRL-68 cells was significantly decreased by OGD treatment, while DEX reversed this phenomenon in a dose-dependent manner. Moreover, DEX reversed OGD-induced inhibition of cell viability (Fig. 1b). Meanwhile, OGD notably induced the apoptosis of WRL-68 cells, while the apoptotic effect of OGD was partially rescued in the presence of DEX (Fig. 1 c). Consistently, OGD treatment significantly inhibited the expression of $\mathrm{Bcl}-2$ but increased the level of Bax in WRL-68 cells, while the effect of OGD on these two proteins was partially reversed by using DEX (Fig. 1d). Furthermore, the levels of IL-1 $\beta$, IL-6, TNF- $\alpha$ in supernatants of WRL-68 cells were increased by OGD, which was significantly rescued by DEX (Fig. 1e). Taken together, DEX inhibited the OGDinduced cell growth inhibition and inflammatory responses.

\section{DEX reversed the expressions of TUG1, miR-194 and SIRT1 in WRL-68 by OGD-induced}

As shown in Fig. 2 a and Fig. 2 c, the expressions of TUG1 and SIRT1 in WRL-68 cells were down-regulated by OGD treatment, while this phenomenon was reversed by DEX. In contrast, OGD-induced upregulation of miR-194 was significantly rescued in the presence of DEX (Fig. 2b). Consistently, DEX significantly reversed the effect of OGD on SIRT1 expression (Fig. 2d). To sum up, DEX might inhibit OGD-induced hepatocyte injury via mediation of TUG1, SIRT1 and miR-194.

\section{The protective effect of DEX against hepatocyte injury was reversed by TUG1 knockdown}

To investigate the correlation between DEX and TUG1 in OGD-induced hepatocyte injury, the qPCR and MTT assay were performed. As indicated in Fig. 3 a, the expression of TUG1 in WRL-68 cells was obviously inhibited by TUG1 shRNA. This data suggested that TUG1 shRNA was successfully transfected into WRL-68 cells. In addition, the viability of OGD-treated WRL-68 was increased by DEX, while TUG1 knockdown notably reversed this phenomenon (Fig. 3b). Moreover, silencing of TUG1 significantly reversed the inhibitory effect of DEX on apoptosis of OGD-treated WRL-68 cells (Fig. 3 c). Furthermore, DEX significantly reversed the effect of OGD on Bax and Bcl-2 expressions, while TUG1 shRNA partially rescued this phenomenon (Fig. 3d). Meanwhile, the levels of IL-1 $\beta$, IL- 6 , TNF- $\alpha$ in supernatants of OGD-treated WRL-68 cells were greatly inhibited by DEX, while the anti-inflammatory effect of DEX was partially restored by TUG1 shRNA (Fig. 3e). All these results indicated that the effect of DEX on hepatocyte injury was reversed by TUG1 knockdown.

\section{TUG1 mediated miR-194/SIRT1 axis in WRL-68 cells}

In order to investigate the mechanism by which TUG1 mediates the progression of OGD-treated hepatocyte injury, bioinformatics analysis was performed. The data suggested that TUG1 had putative binding sites with miR-194, and miR-194 might directly target SIRT1 (Fig. 4 a). For further verification, dualluciferin reporter gene assay was applied. As shown in Fig. 4b, the relative luciferase activity was decreased by miR-194 mimics co-transfected with TUG1-wt or SIRT1-wt, whereas no significant difference was observed with TUG1-mut or SIRT1-mut, suggesting that there were potential binding sites between miR-194 and TUG1, and SIRT1 would be a target gene of miR-194. Furthermore, the qPCR results showed that sh-TUG1 could up-regulate the expression of miR-194 (Fig. 4 c). Then, the expression of miR-194 was increased by miR-194 mimics but reduced in the presence of miR-194 inhibitor, and miR194 could negatively regulate SIRT1 level (Fig. 4d). Moreover, western blotting results showed that miR194 mimics and sh-TUG1 could inhibit the expression of SIRT1 (Fig. 4e). Thereby, the data suggested that TUG1 regulated SIRT1 expression via sponging miR-194.

\section{MiR-194 mimics significantly reversed DEX-induced inhibition of OGD-induced WRL-68 cell injury}

In order to detect the function of miR-194 in DEXmediated hepatocyte injury, MTT was performed. As shown in Fig. 5 a, DEX could increase the viability of OGD-treated WRL-68 cells, while the effect of DEX on cell viability was rescued in the presence of miR-194 mimics. Consistently, miR-194 mimics reversed the antiapoptotic effect of DEX on OGD-treated hepatocytes (Fig. 5b). In addition, DEX significantly up-regulated the level of $\mathrm{Bcl}-2$ but inhibited the expression of $\mathrm{Bax}$ in OGD-treated WRL-68 cells, which was partially reversed by miR-194 mimics (Fig. 5 c). Furthermore, DEXinduced decrease of TNF- $\alpha$, IL- $1 \beta$ and IL- 6 in OGDtreated WRL-68 cells was rescued in the presence of miR-194 mimics (Fig. 5d). In summary, miR-194 mimics reversed the protective effect of DEX against OGDinduced WRL-68 cell injury. 


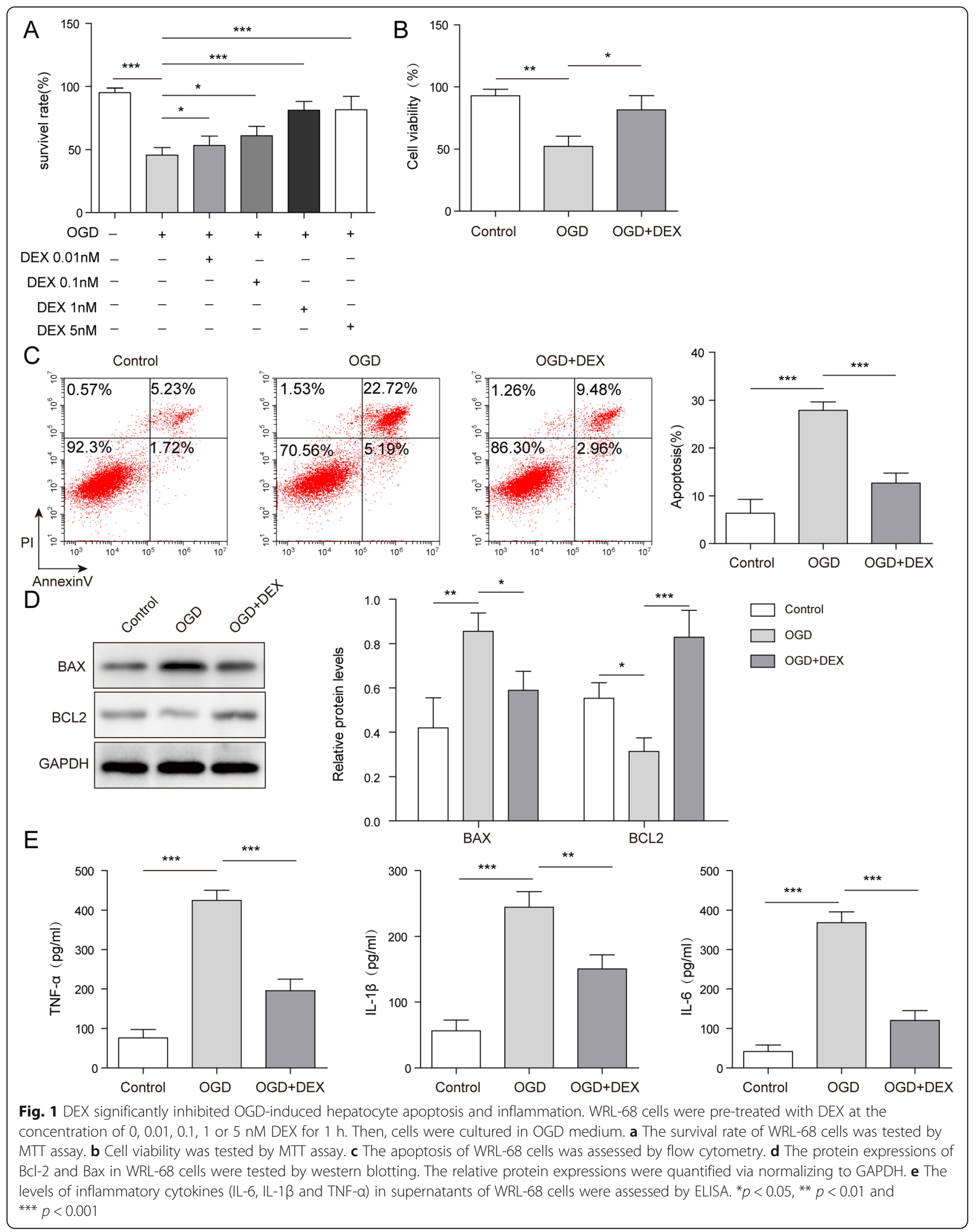



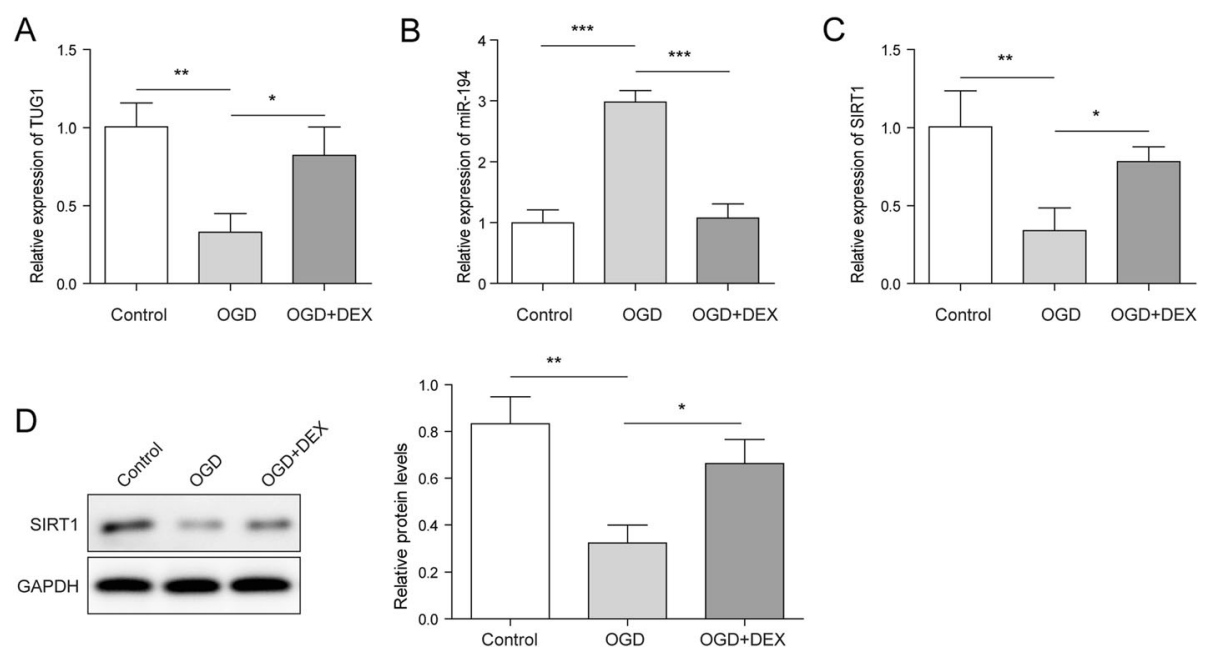

Fig. 2 DEX reversed the expressions of TUG1, miR-194 and SIRT1 in WRL-68 by OGD-induced. WRL-68 cells were pre-treated with 1 nm DEX for $1 \mathrm{~h}$ and then cultured in OGD medium. a The expression of TUG1 in WRL-68 cells was detected by qPCR assay. $\mathbf{b}$ The level of miR-194 in WRL-68 cells was detected by qPCR assay. c The level of SIRT1 in WRL-68 cells was detected by qPCR assay. $\mathbf{d}$ The protein expression of SIRT1 in WRL-68 cells was detected by western blotting. The relative protein expressions were quantified via normalizing to GAPDH. ${ }^{*} p<0.05,{ }^{* *} p<0.01$ and *** $p<0.001$

\section{DEX inhibited OGD-induced hepatocyte injury via mediation of TUG1/miR-194/SIRT1 axis}

To further confirm the mechanism by which DEX mediates OGD-induced hepatocyte injury, MTT assay was used. As shown in Fig. 6 a, DEX could reverse the effect of OGD on cell viability, while this phenomenon was partially reversed by TUG1 silencing. However, the effect of TUG1 knockdown was reversed by miR-194 inhibition or SIRT1 overexpression (Fig. 6 a). In addition, TUG1 shRNA could reverse DEX-induced inhibition of cell apoptosis, which was rescued by miR-194 inhibition or SIRT1 up-regulation (Fig. 6b). Consistently, knocking down TUG1 partially inhibited the effect of DEX on Bax and Bcl-2 expressions in OGD-treated WRL-68 cells, while miR-194 inhibitor or pcDNA3.1-SIRT1 reversed this phenomenon (Fig. 6 c). Finally, silencing of TUG1 up-regulated the levels of TNF- $\alpha$, IL- $1 \beta$ and IL- 6 in DEX and OGD co-treated cell supernatants (Fig. 6d). Nevertheless, the effect of TUG1 shRNA on these inflammatory factors in hepatocytes was reversed when transfected with miR-194 inhibitor or pcDNA3.1-SIRT1 (Fig. 6d). Taken with the above data, DEX inhibited OGD-induced hepatocyte injury by mediation of TUG1/ miR-194/SIRT1 axis.

\section{Discussion}

Prolonged portal vein occlusion can lead to ischemic liver injury in liver transplantation. If the blood supply is restored quickly, cells can be protected against injury. However, improper reperfusion impairs the functions of organs, including liver [23-26]. Ischemia has become a serious obstacle to liver transplantation. It can induce oxygen and nutrient deprivation [27]. The present study firstly found that DEX could reverse OGD-induced hepatocyte injury through regulation of TUG1/miR-194/ SIRT1 signaling pathway, further supplementing the mechanism by which DEX mediated the protection against of liver injury.

It has been previously reported that DEX can act as an anti-inflammatory agent $[28,29]$. For instance, Feng Z et al. found DEX attenuated the inflammatory responses in fatty liver disease [30]. In addition, DEX reduced ventilator-induced inflammation in lung injury via ERK1/2 pathway activation [31]. Meanwhile, it wasreported that DEX could inhibit the liver injury. Tong $\mathrm{F}$ et al. showed that DEX alleviated lipopolysaccharide induced acute liver injury in rats by inhibiting caveolin-1 downstream signaling pathway [32]. Zhou $\mathrm{H}$ et al. found that DEX could inhibit the liver injury though promoting macrophage M2 activation via PPAR $/$ STAT3 signaling [33]. Consistently, our data indicated that DEX reversed OGD-induced inflammatory responses and injury in hepatocytes, suggesting that DEX could act as an inhibitory agent in liver injury. On the other hand, DEX ameliorated liver injury in vivo through $\alpha 2 \mathrm{~A}$ subtype, and the mechanism was due to inhibit TLR4/NF- $\mathrm{kB}$ pathway and reduce the level of inflammatory mediators [34]. Consistently, our study found that DEX suppressed the inflammatory responses in OGD-treated hepatocytes through mediation of TUG1/miR-194/SIRT1 axis. Thus, the protective functions of DEX on liver injury could regulate the different mechanisms between in vitro and in vivo study. Intriguingly, it was reported that $30 \mu \mathrm{g} / \mathrm{kg}$ improved LPS-induced acute liver injury in SD rats [35]. 


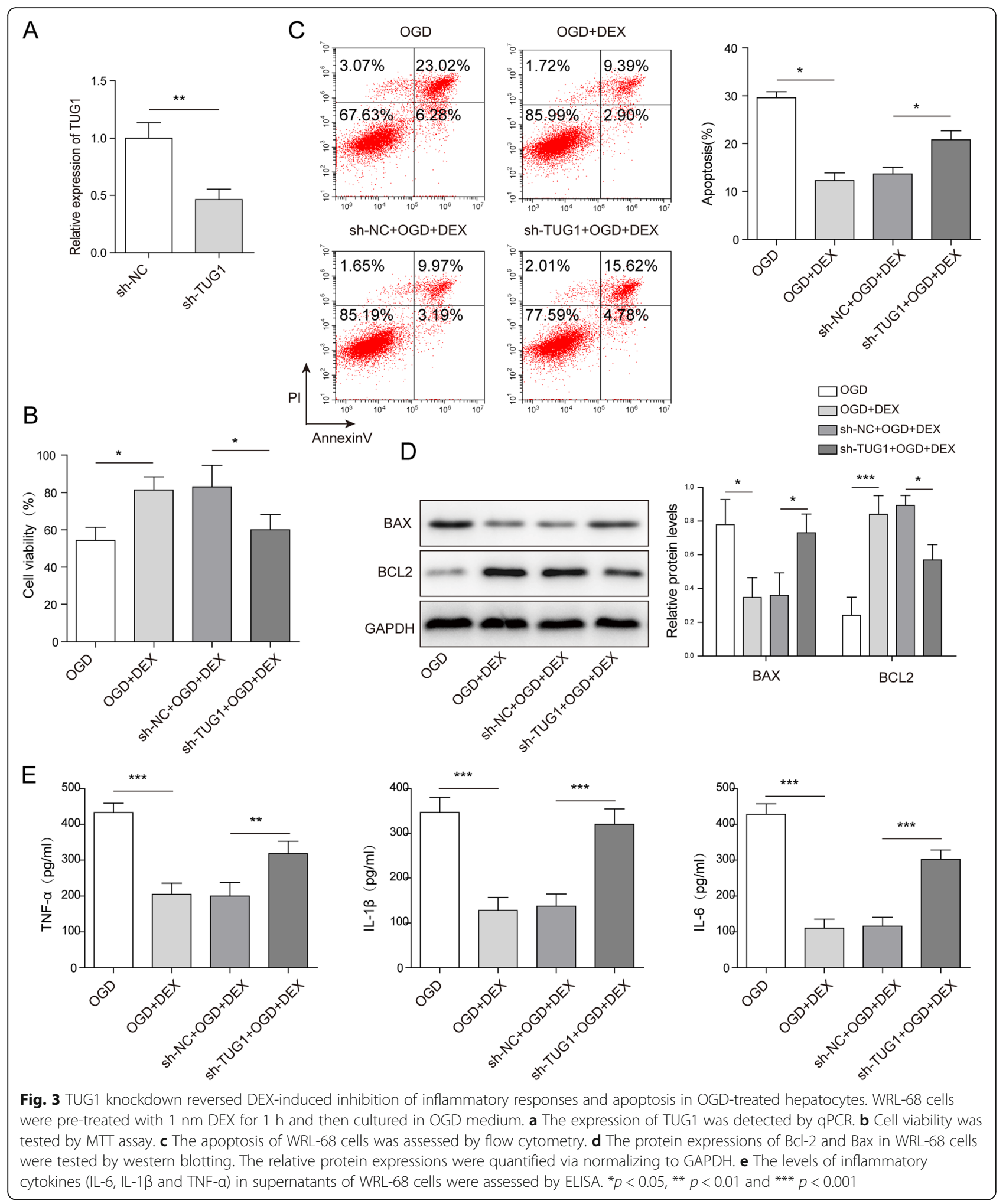




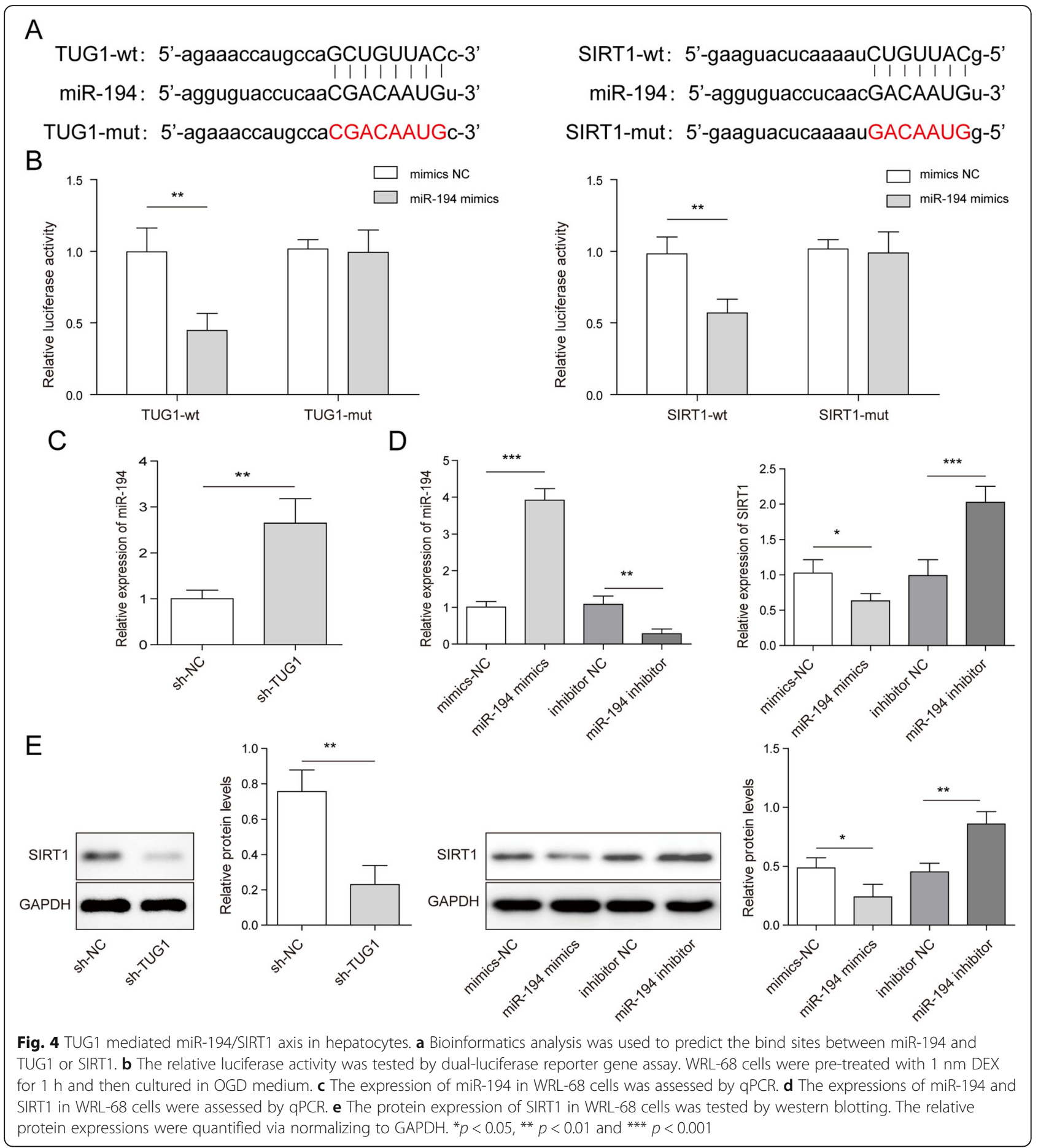

Saleh et al. found that continuous use of $0.8 \mu \mathrm{g} / \mathrm{kg} / \mathrm{h}$ DEX during the operation could protect the ischemia-reperfusion injury and enhance the liver function after adult liver transplantation [36]. However, in our study indicated that $1 \mathrm{~nm}$ DEX inhibited ODG-induced hepatocytes proliferation and promoted apoptosis, and this concentration also used in many researches [37].
It has been reported that TUG1 plays important roles in cell injury and inflammation $[38,39]$. In addition, TUG1 has been found to relieve the liver injury [40]. Our finding was consistent to this previous report, confirming that TUG1 could serve as a mediator in liver injury. Meanwhile, our study found that miR-194/SIRT1 axis could be regulated by TUG1, while Zhang $\mathrm{H}$ et al. indicated that TUG1 could inhibit LPS-induced 


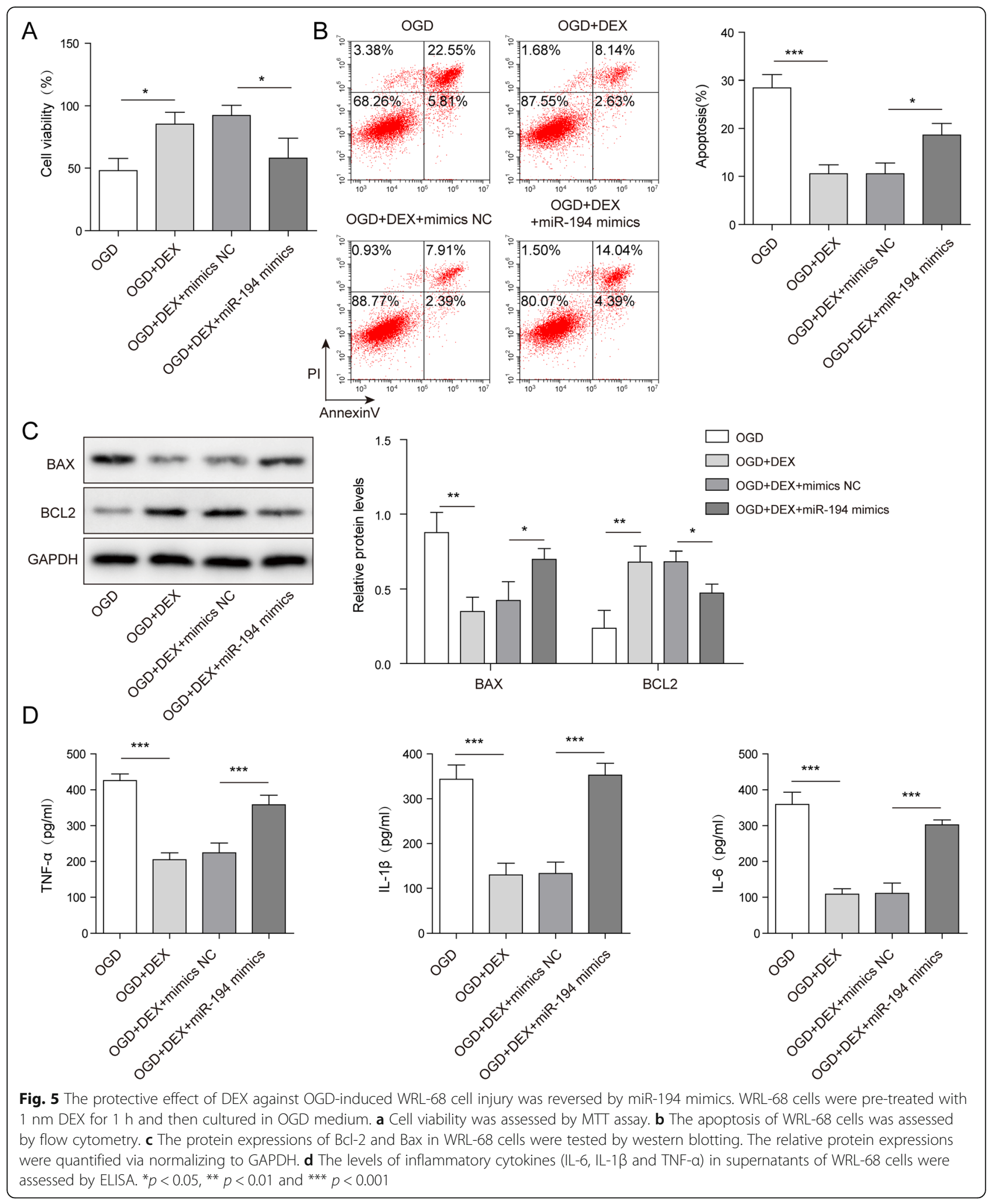




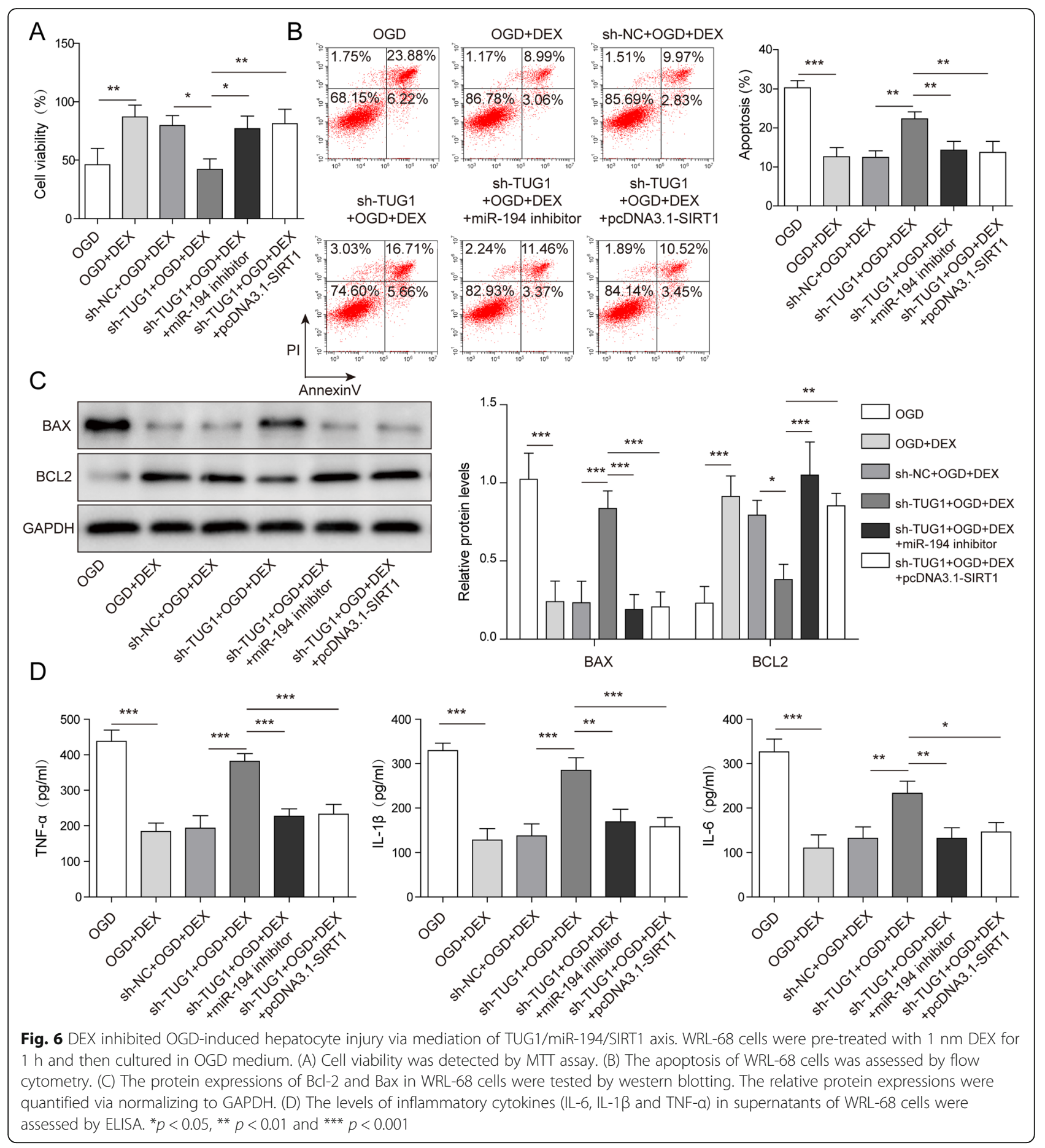

apoptosis and inflammatory response though downregulation of miR-29b, NF- $\mathrm{kB}$ and JAK/STAT pathways [14]. In our study, we found that DEX increased TUG1 expression in OGD-induced WRL-68, however, knockdown of TUG1 reversed the protective effect of DEX in OGD-induced WRL-68 cells, suggesting DEX played a key role in liver injury by regulating the expression of
TUG1. Thus, more mechanisms by which TUG1 regulates liver injury need to be explored in the future.

Recently, serum liver-cell-derived microRNAs (HDmiRs) were considered as early, stable, sensitive and specific biomarkers for liver injury [41]. Meanwhile, miR-194 is known to be a crucial mediator in inflammatory responses $[42,43]$. Moreover, miR-194 could aggravate the liver injury [44]. 
Consistently, this research found that miR-194 was up-regulated in OGD-treated hepatocyte injury, and miR-194 overexpression restored the regulatory effect of DEX on apoptosis and inflammation in OGDinduced WRL-68 cells. In addition, miR-194 was overexpressed in patients with liver diseases, and Yangonin inhibited cell aging by regulating miR-194 and alleviated alcohol-induced liver injury [44]. Our research was similar to this study, indicating that miR194 could be sponged by TUG1 in hepatocyte injury. Taken with the above results, miR-194 could act as an important mediator of liver function.

SIRT1, an $\mathrm{NAD}^{+}$dependent type III histone/protein deacetylase, plays a key role in protecting against cellular stress and in controlling metabolic pathways which are critical to ischemia/hypoxia $[45,46]$. In addition, previous studies found SIRT1 inhibited inflammatory responses in heart, liver, brain and kidney injury [47, 48]. Meanwhile, our research indicated that SIRT1 overexpression could reverse the pro-inflammatory effect of sh-TUG1 in hepatocytes. Thus, our data were in line with the function of SIRT1, confirming that TUG1 mediated the inflammatory responses via indirectly targeting SIRT1. On the other hand, NLRP3 is known to be an inflammasome, and it can promote the levels of inflammatory cytokines in organ respiration $[49,50]$. It was found that SIRT1 inhibited the activation of NLRP3 and the secretion of IL- $1 \beta$ in cerebral ischemia [51]. In present study, our findings revealed that SIRT1 could act as an inflammatory suppressor in progression of hepatocytes injury. Taken together, DEX could inhibit OGD-induced hepatocyte apoptosis and inflammation via regulating of TUG1/miR-194/SIRT1 axis.

\section{Conclusions}

In the present study, in vitro data showed that DEX could protect against OGD-induced hepatocyte injury. In addition, DEX reversed OGD-induced hepatocyte apoptosis and inflammation through regulation of TUG1, miR-194 and SIRT1. The protective effect of DEX on OGD-treated hepatocytes was accomplished through TUG1/miR-194/SIRT1 signaling pathway. These results might provide a scientific basis for the application of DEX on treatment of liver injury. However, more randomized controlled trials were required to verify the effect of DEX in clinic.

\footnotetext{
Abbreviations

DEX: Dexmedetomidine hydrochloride; OGD: Oxygen-glucose deprivation; TUG1: Taurine up-regulated gene 1; LncRNAs: Long non-coding RNAs; SIRT1: Sirtuin 1; SDS-PAGE: Sodium dodecyl sulfate-polyacrylamide gel electrophoresis; PVDF: Polyvinylidene fluoride; DMSO: Dimethyl sulfoxide; FACS: Fluorescence activated Cell Sorting; LSD: Least significant difference; MTT: 3-(4,5-dimethyl thiazol-2-yl)-5-diphenyl tetrazolium bromide; NC: Negative control; SD: Standard deviations
}

\section{Acknowledgements}

We would like to give our sincere gratitude to the reviewers for their constructive comments.

\section{Authors' contributions}

Conception and study design: XXG; Data acquisition: XXX, HHL; Data analysis: RNW, WMH; Manuscript drafting: LXC; Manuscript revising: YWL, JM. All authors have read and approved the final version of this manuscript to be published.

\section{Funding}

This work was supported by Zhanjiang Science and Technology Special Competitive Distribution Project (2015A01021).

\section{Availability of data and materials}

All data generated or analyzed during this study are included in this article. The datasets used and/or analyzed during the current study are available from the corresponding author on reasonable request.

\section{Declarations}

\section{Ethics approval and consent to participate}

Not Applicable. This article does not contain any studies with human participants or animals performed by any of the authors.

\section{Consent for publication}

Not Applicable. This article does not contain any studies with human participants performed by any of the authors.

\section{Competing interests}

The authors declare that there is no conflict of interest.

\section{Author details}

'Department of Anesthesiology, Affiliated Hospital of Guangdong Medical University, No.57, South People's Avenue, Xiashan District, 524001 Zhanjiang, Guangdong Province, P.R. China. ${ }^{2}$ Operating room, Affiliated Hospital of Guangdong Medical University, 524001 Zhanjiang, Guangdong Province, P.R. China. ${ }^{3}$ Department of Thyroid and Breast Surgery, Affiliated Hospital of Guangdong Medical University, 524001 Zhanjiang, Guangdong Province, P.R. China.

Received: 22 December 2020 Accepted: 6 May 2021

Published online: 26 May 2021

\section{References}

1. Kim MH, Kim HH, Jeong JM, Shim YR, Lee JH, Kim YE, et al. Ginsenoside F2 attenuates chronic-binge ethanol-induced liver injury by increasing regulatory T cells and decreasing Th17 cells. J Ginseng Res. 2020;44(6):815-22.

2. Mullins C, Beaulac K, Sylvia L. Reply. Drug-Induced Liver Injury (DILI) With Micafungin: The Importance of Causality Assessment. Ann Pharmacother. 2020:1060028020973607

3. Dixon J, Lane K, Macphee I, Philips B. Xenobiotic metabolism: the effect of acute kidney injury on non-renal drug clearance and hepatic drug metabolism. Int J Mol Sci. 2014:15(2):2538-53.

4. Jaeschke H, Duan L, Nguyen N, Ramachandran A. Mitochondrial Damage and Biogenesis in Acetaminophen-induced Liver Injury. Liver Res. 2019;3(34):150-6.

5. Wang ZX, Huang CY, Hua YP, Huang WQ, Deng LH, Liu KX Dexmedetomidine reduces intestinal and hepatic injury after hepatectomy with inflow occlusion under general anaesthesia: a randomized controlled trial. Br J Anaesth. 2014;112(6):1055-64.

6. Zhang X, Wang J, Qian W, Zhao J, Sun L, Qian Y, et al. Dexmedetomidine inhibits tumor necrosis factor-alpha and interleukin 6 in lipopolysaccharidestimulated astrocytes by suppression of c-Jun $\mathrm{N}$-terminal kinases. Inflammation. 2014:37(3):942-9.

7. Zhao Y, Kong GY, Pei WM, Zhou B, Zhang QQ, Pan BB. Dexmedetomidine alleviates hepatic injury via the inhibition of oxidative stress and activation of the Nrf2/HO-1 signaling pathway. Eur Cytokine Netw. 2019;30(3):88-97.

8. Mattick JS, Makunin IV. Non-coding RNA. Human molecular genetics. 2006; 15 Spec No 1:R17-29. 
9. Schonrock N, Harvey RP, Mattick JS. Long noncoding RNAs in cardiac development and pathophysiology. Circ Res. 2012;111(10):1349-62.

10. Barry G, Briggs JA, Vanichkina DP, Poth EM, Beveridge NJ, Ratnu VS, et al. The long non-coding RNA Gomafu is acutely regulated in response to neuronal activation and involved in schizophrenia-associated alternative splicing. Mol Psychiatry. 2014;19(4):486-94.

11. Prensner JR, lyer MK, Balbin OA, Dhanasekaran SM, Cao Q, Brenner JC, et al. Transcriptome sequencing across a prostate cancer cohort identifies PCAT1, an unannotated lincRNA implicated in disease progression. Nature Biotechnology. 2011;29(8):742-9.

12. Zhang Q, Geng PL, Yin P, Wang XL, Jia JP, Yao J. Down-regulation of long non-coding RNA TUG1 inhibits osteosarcoma cell proliferation and promotes apoptosis. Asian Pac J Cancer Prev. 2013;14(4):2311-5.

13. Han Y, Liu Y, Gui Y, Cai Z. Long intergenic non-coding RNA TUG1 is overexpressed in urothelial carcinoma of the bladder. J Surgical Oncol. 2013;107(5):555-9.

14. Zhang H, Li H, Ge A, Guo E, Liu S, Zhang L. Long non-coding RNA TUG1 inhibits apoptosis and inflammatory response in LPS-treated H9c2 cells by down-regulation of miR-29b. Biomedicine \& pharmacotherapy = Biomedecine \& pharmacotherapie. 2018;101:663-9.

15. Heneghan HM, Miller N, Kerin MJ. MiRNAs as biomarkers and therapeutic targets in cancer. Curr Opin Pharmacol. 2010;10(5):543-50.

16. Viegas ATB, Guedes JR, Oliveira AR, Cardoso AMS, Cardoso ALC. miRNAs: New Biomarkers and Therapeutic Targets in Dementia. Curr Pharm Design. 2017;23(5):669-92.

17. Venugopal SK, Jiang J, Kim TH, Li Y, Wang SS, Torok NJ, et al. Liver fibrosis causes downregulation of miRNA-150 and miRNA-194 in hepatic stellate cells, and their overexpression causes decreased stellate cell activation. Am J Physiology Gastrointestinal Liver Physiology. 2010;298(1):G101-6.

18. Wu JC, Chen R, Luo X, Li ZH, Luo SZ, Xu MY. MicroRNA-194 inactivates hepatic stellate cells and alleviates liver fibrosis by inhibiting AKT2. World Gastroenterology. 2019;25(31):4468-80.

19. Ogiku M, Kono H, Hara M, Tsuchiya M, Fujii H. Glycyrrhizin prevents liver injury by inhibition of high-mobility group box 1 production by Kupffer cells after ischemiareperfusion in rats. J Pharmacol Exp Ther. 2011;339(1):93-8.

20. Zhao X, Jin Y, Li L, Xu L, Tang Z, Qi Y, et al Corrigendum to "MicroRNA-128$3 p$ aggravates doxorubicin-induced liver injury by promoting oxidative stress via targeting Sirtuin-1" [Pharmacol. Res. 146 (2019) 104276]. Pharmacol Res. 2020;151:104474.

21. Bai $T$, Yang $Y$, Yao $Y L$, Sun $P$, Lian $L H, W u ~ Y L$, et al. Betulin alleviated ethanol-induced alcoholic liver injury via SIRT1/AMPK signaling pathway. Pharmacol Res. 2016;105:1-12.

22. Ren $Y L$, Zhang W. Propofol promotes apoptosis of colorectal cancer cells via alleviating the suppression of IncRNA HOXA11-AS on miRNA let-7i. Biochemistry and cell biology = Biochimie. et biologie cellulaire. 2020;98(2):90-8.

23. Granger DN, Kvietys PR. Reperfusion injury and reactive oxygen species: The evolution of a concept. Redox Biol. 2015;6:524-51.

24. Kupiec-Weglinski JW, Busuttil RW. Ischemia and reperfusion injury in liver transplantation. Transplantation proceedings. 2005;37(4):1653-6.

25. Zhu YX, Zhou JH, Li GW, Zhou WY, Ou SS, Xiao XY. Dexmedetomidine protects liver cell line L-02 from oxygen-glucose deprivation-induced injury by down-regulation of microRNA-711. Eur Rev Med Pharmacol Sci. 2018; 22(19):6507-16.

26. Honda M, Takeichi T, Asonuma K, Tanaka K, Kusunoki M, Inomata Y. Intravital imaging of neutrophil recruitment in hepatic ischemia-reperfusion injury in mice. Transplantation. 2013;95(4):551-8.

27. Xu J, Zang Y, Liu D, Yang T, Wang J, Wang Y, et al. DRAM is Involved in Hypoxia/Ischemia-Induced Autophagic Apoptosis in Hepatocytes. Aging Dis. 2019;10(1):82-93.

28. Li H, Lu C, Yao W, Xu L, Zhou J, Zheng B. Dexmedetomidine inhibits inflammatory response and autophagy through the circLrp1b/miR-27a-3p/Dram2 pathway in a rat model of traumatic brain injury. Aging (Albany NY). 2020;12.

29. Wang QS, Xu BX, Fan KJ, Li YW, WU J, Wang TY. Dexamethasone-Loaded Thermosensitive Hydrogel Suppresses Inflammation and Pain in CollagenInduced Arthritis Rats. Drug Des Devel Ther. 2020;14:4101-13.

30. Feng Z, Pang $L$, Chen S, Pang X, Huang Y, Qiao Q, et al. Didymin ameliorates dexamethasone-induced non-alcoholic fatty liver disease by inhibiting TLR4/NF-kappaB and PI3K/Akt pathways in C57BL/6J mice. Int Immunopharmacol. 2020;88:107003.

31. Zhu CH, Yu J, Wang BQ, Nie Y, Wang L, Shan SQ. Dexmedetomidine reduces ventilator-induced lung injury via ERK1/2 pathway activation. Mo Med Rep. 2020;22(6):5378-84
32. Tong F, Shen W, Song P, Song J, Hu Y, Liu F, et al. Dexmedetomidine attenuates lipopolysaccharide induced acute liver injury in rats by inhibiting caveolin-1 downstream signaling pathway. Biosci Rep. 2021.

33. Zhou H, Sun J, Zhong W, Pan X, Liu C, Cheng F, et al. Dexmedetomidine preconditioning alleviated murine liver ischemia and reperfusion injury by promoting macrophage M2 activation via PPARgamma/STAT3 signaling. Int Immunopharmacol. 2020;82:106363.

34. Wang Y, Wu S, Yu X, Zhou S, Ge M, Chi X, et al. Dexmedetomidine Protects Rat Liver against Ischemia-Reperfusion Injury Partly by the alpha2AAdrenoceptor Subtype and the Mechanism Is Associated with the TLR4/NFkappaB Pathway. Int J Mol Sci. 2016;17(7).

35. Sha J, Zhang H, Zhao Y, Feng X, Hu X, Wang C, et al. Dexmedetomidine attenuates lipopolysaccharide-induced liver oxidative stress and cell apoptosis in rats by increasing GSK-3beta/MKP-1/Nrf2 pathway activity via the alpha2 adrenergic receptor. Toxicol Appl Pharmacol. 2019;364:144-52.

36. Fayed NA, Sayed El, Saleh SM, Ehsan NA, Elfert AY. Effect of dexmedetomidine on hepatic ischemia-reperfusion injury in the setting of adult living donor liver transplantation. Clin Transplant. 2016;30(4):470-82.

37. Cui J, Zhao H, Yi B, Zeng J, Lu K, Ma D. Dexmedetomidine Attenuates Bilirubin-Induced Lung Alveolar Epithelial Cell Death In Vitro and In Vivo. Crit Care Med. 2015;43(9):e356-68.

38. Wang W, Zhang S, Yang F, Xie J, Chen J, Li Z. Diosmetin alleviates acute kidney injury by promoting the TUG1/Nrf2/HO-1 pathway in sepsis rats. Int Immunopharmacol. 2020;88:106965.

39. Song T, Wang P, Xin L. LncRNA TUG1 Contributes to Hypoxia-Induced Myocardial Cell Injury Through Downregulating miR-29a-3p in AC16 Cells. J Cardiovasc Pharmacol. 2020;76(5):533-9.

40. Su S, Liu J, He K, Zhang M, Feng C, Peng F, et al. Overexpression of the long noncoding RNA TUG1 protects against cold-induced injury of mouse livers by inhibiting apoptosis and inflammation. FEBS J. 2016;283(7):1261-74.

41. Farid WR, Pan Q, van der Meer AJ, de Ruiter PE, Ramakrishnaiah V, de Jonge J, et al. Hepatocyte-derived microRNAs as serum biomarkers of hepatic injury and rejection after liver transplantation. Liver transplantation: official publication of the American Association for the Study of Liver Diseases and the International Liver Transplantation Society. 2012;18(3):290-7.

42. Cheng L, Tu C, Min Y, He D, Wan S, Xiong F. MiR-194 targets Runx1/Akt pathway to reduce renal fibrosis in mice with unilateral ureteral obstruction. Int Urol Nephrol. 2020;52(9):1801-8.

43. Chen R, Xie F, Zhao J, Yue B. Suppressed nuclear factor-kappa B alleviates lipopolysaccharide-induced acute lung injury through downregulation of CXCR4 mediated by microRNA-194. Respir Res. 2020;21(1):144.

44. Dong R, Wang X, Wang L, Wang C, Huang K, Fu T, et al. Yangonin inhibits ethanol-induced hepatocyte senescence via miR-194/FXR axis. Eur J Pharmacol. 2020:173653.

45. Kim HJ, Joe Y, Yu JK, Chen Y, Jeong SO, Mani N, et al. Carbon monoxide protects against hepatic ischemia/reperfusion injury by modulating the miR34a/SIRT1 pathway. Biochim Biophys Acta. 2015;1852(7):1550-9.

46. Zhang F, Li ZL, Xu XM, Hu Y, Yao JH, Xu W, et al. Protective effects of icariinmediated SIRT1/FOXO3 signaling pathway on intestinal ischemia/ reperfusion-induced acute lung injury. Mol Med Rep. 2015;11(1):269-76.

47. Rickenbacher A, Jang JH, Limani P, Ungethum U, Lehmann K, Oberkofler CE, et al. Fasting protects liver from ischemic injury through Sirt1-mediated downregulation of circulating HMGB1 in mice. J Hepatology. 2014;61(2):301-8.

48. Ren J, Fan C, Chen N, Huang J, Yang Q. Resveratrol pretreatment attenuates cerebral ischemic injury by upregulating expression of transcription factor Nrf2 and HO-1 in rats. Neurochem Res. 2011;36(12):2352-62.

49. He BC, Zhou W, Rui YW, Liu L, Chen B, Su X. MicroRNA-574-5p Attenuates Acute Respiratory Distress Syndrome by Targeting HMGB1. Am J Respir Cell Mol Biol. 2020.

50. Su Y, Zhang Y, Hu Z, He L, Wang W, Xu J, et al. Prokineticin 2 via CalciumSensing Receptor Activated NLRP3 Inflammasome Pathway in the Testicular Macrophages of Uropathogenic Escherichia coli-Induced Orchitis. Front Immunol. 2020;11:570872.

51. Zhang $S$, Jiang L, Che F, Lu Y, Xie Z, Wang H. Arctigenin attenuates ischemic stroke via SIRT1-dependent inhibition of NLRP3 inflammasome. Biochem Biophys Res Commun. 2017;493(1):821-6.

\section{Publisher's Note}

Springer Nature remains neutral with regard to jurisdictional claims in published maps and institutional affiliations. 